\title{
Price Regulation Mechanism of Travelers' Travel Mode Choice in the Driverless Transportation Network
}

\author{
Jianhui Wu, ${ }^{1}$ Yuanfa Ji, ${ }^{2}$ Xiyan Sun $\mathbb{D}^{2},{ }^{2}$ and Yan $X u^{1}$ \\ ${ }^{1}$ School of Information Science and Technology, Hunan Institute of Science and Technology, Yueyang 414006, China \\ ${ }^{2}$ Guangxi Key Laboratory of Precision Navigation Technology and Application, Guilin University of Electronic Technology, \\ Guilin 541004, China \\ Correspondence should be addressed to Xiyan Sun; sunxiyan1@163.com
}

Received 22 February 2020; Revised 27 April 2020; Accepted 12 May 2020; Published 28 May 2020

Academic Editor: Weiwei Qi

Copyright (c) 2020 Jianhui Wu et al. This is an open access article distributed under the Creative Commons Attribution License, which permits unrestricted use, distribution, and reproduction in any medium, provided the original work is properly cited.

To study the guidance method of driverless travel mode choice from the perspective of traffic supply-demand, we assume that all vehicles are driverless and establish a multimodal travel market model to depict the supply-demand relationship of multimodal driverless transportation network. To regulate the disequilibrium multimodal travel market, an optimal price regulation law is proposed, which aims to minimize the supply-demand deviation and the amplitude of price regulation. Then, the existence, uniqueness, and stability of the optimal price regulation law are confirmed. In the calculation process of a numerical example, the travel prices of driverless car and driverless subway are realized by congestion fee and subway fare, respectively. The results indicate that the optimal price regulation law can reduce the supply-demand deviation of the multimodal travel market and guide travelers to choose a reasonable travel mode to travel in the driverless transportation network.

\section{Introduction}

With the development of driverless technology, the city is again at the crossroads of a historic transformation in the transportation technology. Hence, it is important to determine how the impact of driverless technology on urban transportation should be addressed. Such problems have attracted considerable attention from scholars. Morando et al. [1] believed that the high penetration rate of autonomous vehicles can significantly improve the traffic safety in urban transportation network. Ye and Yamamoto [2] pointed out that the advantages of setting dedicated lanes for automatic vehicles are significant when automatic vehicles are in the medium density range of mixed traffic flow. Smolnicki and Sołtys [3] investigated the effects of different driverless mobility solutions on urban spatial structures. Zhang et al. [4] studied the impact of a shared autonomous vehicles system on urban parking demand. Kamel et al. [5] analyzed the effects of user preferences on the modal split of shared autonomous vehicles. Yi et al. [6] studied the effect of the ambient temperature on the energy cost and charging demand for autonomous electric vehicles. Moreno et al. [7] believed that ride sharing can more effectively reduce extra vehicle kilometers, while the same amount of trips by shared autonomous vehicles will require longer empty trips.

Currently, research on urban traffic planning and management in the driverless environment focuses primarily on road planning, parking planning, public transport planning, and traffic management planning. The purpose of road planning is to solve the driving problem of driverless vehicles on the road. Relevant achievements include lane planning (e.g., Liu and Song [8] and and Xia et al. [9]), traffic signal control planning (e.g., Domínguez and Sanguino [10] and Jiang [11]), and speed limit planning (e.g., Tajalli and Hajbabaie [12] and 
Liu et al. [13]). The purpose of parking planning is to solve the parking problem of driverless vehicles, and relevant achievements include parking lot design (e.g., Nourinejad et al. [14] and Estepa et al. [15]) and parking lot management (e.g., Yamashita and Takami [16] and Wang et al. [17]). The purpose of public transport planning is to attract some travelers to travel by the large capacity and high occupancy of driverless public transport. Relevant achievements include driverless public transport type planning (e.g., Leich and Bischoff [18] and Abe [19]) and driverless public transport line design (e.g., Cao and Ceder [20] and Tong et al. [21]). The purpose of traffic management planning is to maintain traffic order and ensure smooth traffic and safe run. Relevant achievements include vehicles' safety performance (e.g., Ye and Yamamoto [22] and Virdi et al. [23]), traffic laws and regulations (e.g., Prakken [24] and Bartolini et al. [25]), and traffic control (e.g., Wagner [26] and Tettamanti et al. [27]).

Overall, the new driverless technology has brought great changes to urban transportation system. However, the driverless transportation system is large and complex. There may be congestion on some roads during the peak period, and it may be difficult to find a parking place in office and residential areas during the nonpeak period. Kitamura et al. [28] pointed out that traffic planners need to find effective ways to induce or suppress travel demand in the next generation of transportation planning methodologies. Brideges [29] believed that the "multitraveler sharing" public transport system can solve the problem of urban congestion and proposed a "carrot and stick" mechanism to encourage travelers to share their vehicles with others. Therefore, it is also necessary to strengthen the guidance of travelers' travel mode choice in the driverless transportation network.

Ye and Wang [30] proposed a bilevel programming model of congestion pricing for two travel modes: driverless vehicle and conventional vehicle. Zhang et al. [31] studied the integrated morning-evening commuting pattern at the system optimum in the fully autonomous vehicle environment. On the whole, there are few researchers to study the guidance method of driverless travel mode choice from the relationship of traffic supply-demand. However, the disequilibrium of traffic supply-demand is the fundamental reason for urban traffic congestion. Based on this, this study regards each type of driverless travel modes on each OD pair as a travel market, regards driving time, driving cost, and perceived traffic service quality as travel price, and attempts to seek the price regulation mechanism of multidriverless travel mode in terms of market supplydemand.

The remainder of this paper is organized as follows. In Section 2, we establish a multimodal travel market model. In Section 3, we present an optimal price regulation law of disequilibrium multimodal travel market and prove the existence, uniqueness, and stability of the optimal price regulation law. A numerical example is demonstrated in Section 4, and the conclusions are presented in Section 5.

\section{Multimodal Travel Market Model}

To depict the supply-demand relationship of the multimodal driverless transportation network, we developed a multimodal travel market model. In the driverless transportation network, we suppose that all travelers do not have a private car, and they can only travel by driverless vehicles. Furthermore, we assume that there are $M$ types of driverless travel modes (e.g., driverless car, driverless bus, and driverless subway) on OD pair $(i, j)$. If we regard each type of driverless travel mode as a travel market, then there are $M$ types of travel markets on OD pair $(i, j)$.

2.1. Notional Supply and Demand. We define all travelers of the $m^{\text {th }}$ travel mode who need to travel from the origin node $i$ to the destination node $j$ as the demand side of the $m^{\text {th }}$ travel market. According to the definition of the demand function in the elastic demand traffic assignment problem, we define the linear combination between exogenous variable vector $X_{i j}^{m}$ and travel price $P_{i j}^{m}$ as the notional demand $\widetilde{D}_{i j}^{m}$ of the $m^{\text {th }}$ travel market on OD pair $(i, j)$ at the $k^{\text {th }}$ period. It can be expressed as

$$
\begin{aligned}
\widetilde{D}_{i j}^{m}(k)=\alpha_{i j}^{m}\left\{X_{i j}^{m}(k)\right\}^{T}+\beta_{i j}^{m} P_{i j}^{m}(k), \\
\forall \beta_{i j}^{m}<0, m \in M_{i j}, i, j \in N, i \neq j,
\end{aligned}
$$

where $\alpha_{i j}^{m}$ represents the influence coefficient vector of $X_{i j}^{m}$ on $\widetilde{D}_{i j}^{m}, \beta_{i j}^{m}$ is the influence coefficient of $P_{i j}^{m}$ on $\widetilde{D}_{i j}^{m}, X_{i j}^{m}$ reflects the total travel demand, as well as the traveler's gender, age, occupation, income, etc., $\left\{X_{i j}^{m}(k)\right\}^{T}$ is the transpose of the vector $X_{i j}^{m}, P_{i j}^{m}$ is a comprehensive index that reflects the driving time, driving cost, perceived traffic service quality, etc., $M_{i j}$ is the set of all travel modes on OD pair $(i, j)$, and $N$ is the set of all nodes.

We define the transport managers who can provide transport services to the demand-side of the $m^{\text {th }}$ travel market on OD pair $(i, j)$ in the short period as the supply side of the $m^{\text {th }}$ travel market. Considering that the supply of driverless car (or driverless bus, driverless subway, etc.) in the short period is affected a little by travel price, we ignore the influence of travel price. Suppose that the notional supply $\widetilde{S}_{i j}^{m}$ of the $m^{\text {th }}$ travel market on OD pair $(i, j)$ at the $k^{\text {th }}$ period is only related to the exogenous variable vector $Y_{i j}^{m}$. It can be expressed as

$$
\widetilde{S}_{i j}^{m}(k)=\eta_{i j}^{m}\left\{Y_{i j}^{m}(k)\right\}^{T}, \quad \forall m \in M_{i j}, i, j \in N, i \neq j,
$$

where $\eta_{i j}^{m}$ represents the influence coefficient vector of $Y_{i j}^{m}$ on $\widetilde{S}_{i j}^{m}$ and $Y_{i j}^{m}$ reflects the total supply, severe weather, natural disaster, traffic accident, etc. 
2.2. Effective Supply and Demand. Suppose that the supply side between different travel modes is independent of each other, and the excessive demand of one travel market may spill over the effective demand of other travel market in the multimodal travel market. For example, if the demand of the driverless car travel market exceeds the supply, some driverless car travelers may switch to other driverless travel modes, such as driverless bus or driverless subway. Referring to the relevant research results in the field of economics (Browne [32]), we define the linear combination between the notional demand of the $m^{\text {th }}$ travel market and the effective supply-demand difference of other travel markets as the effective demand $D_{i j}^{m}$ of the $m^{\text {th }}$ travel market on OD pair $(i, j)$ at the $k^{\text {th }}$ period. It can be expressed as

$$
\begin{array}{r}
D_{i j}^{m}(k)=\widetilde{D}_{i j}^{m}(k)+\sum_{r \neq m} \varphi_{i j}^{r}\left[D_{i j}^{r}(k)-S_{i j}^{r}(k)\right], \\
\forall \varphi_{i j}^{r} \geq 0, r \in M_{i j} .
\end{array}
$$

Applying formula (1) into (3), we have

$$
\begin{aligned}
D_{i j}^{m}(k)= & \alpha_{i j}^{m}\left\{X_{i j}^{m}(k)\right\}^{T}+\beta_{i j}^{m} P_{i j}^{m}(k) \\
& +\sum_{r \neq m} \varphi_{i j}^{r}\left[D_{i j}^{r}(k)-S_{i j}^{r}(k)\right],
\end{aligned}
$$

where $\varphi_{i j}^{r}$ represents the spillover effect coefficient between the $m^{\text {th }}$ travel market and the $r^{\text {th }}$ travel market on OD pair $(i, j)$.

Considering the interaction of the effective supply between different OD pairs in the same travel mode (e.g., a driverless bus passes through node $i$ and node $i^{\prime}$ to node $j$; if the effective demand on OD pair $(i, j)$ increases, the effective supply on OD pair $(i, j)$ will also increase, and the effective supply on OD pair $\left(i^{\prime}, j\right)$ will decrease), we define the linear combination between the notional demand of the $m^{\text {th }}$ travel market and the effective supply of other travel markets as the effective supply $S_{i j}^{m}$ of the $m^{\text {th }}$ travel market on OD pair $(i, j)$ at the $k^{\text {th }}$ period. It can be expressed as

$$
S_{i j}^{m}(k)=\widetilde{S}_{i j}^{m}(k)+\sum_{i^{\prime} \neq i \text { or } j^{\prime} \neq j} \vartheta_{i j-i^{\prime} j}^{m} S_{i^{\prime} j}^{m}(k), \quad \forall \vartheta_{i^{\prime} j}^{m} \leq 0, i^{\prime}, j^{\prime} \in N .
$$

Applying formula (2) into (5), we have

$$
S_{i j}^{m}(k)=\eta_{i j}^{m}\left\{Y_{i j}^{m}(k)\right\}^{T}+\sum_{i^{\prime} \neq i \text { or } j^{\prime} \neq j} \vartheta_{i j-i^{\prime} j}^{m} S_{i^{\prime} j}^{m}(k),
$$

where $\vartheta_{i j-i^{\prime} j}^{m}$ represents the influence coefficient of the $m^{\text {th }}$ travel market between OD pair $(i, j)$ and OD pair $\left(i^{\prime}, j^{\prime}\right)$ and $\vartheta_{i^{\prime} j}^{m}=0$ indicates that the $m^{\text {th }}$ travel markets between OD pair $(i, j)$ and OD pair $\left(i^{\prime}, j^{\prime}\right)$ are independent of each other.
2.3. Model Formulation. In the multimodal travel market, if the effective demand $D_{i j}^{m}$ is less than the effective supply $S_{i j}^{m}$, the travel market is in the state of oversupply and the market trading volume $Q_{i j}^{m}=D_{i j}^{m}$; if the effective demand $D_{i j}^{m}$ is more than the effective supply $S_{i j}^{m}$, the travel market is in the state of overdemand and the market trading volume $Q_{i j}^{m}=S_{i j}^{m}$; if the effective demand $D_{i j}^{m}$ is equal to the effective supply $S_{i j}^{m}$, the travel market is in the state of equilibrium and the market trading volume $Q_{i j}^{m}=$ $D_{i j}^{m}=S_{i j}^{m}$. Clearly, the multimodal travel market trading follows the principle of short side. The trading volume $Q_{i j}^{m}$ of the $m^{\text {th }}$ travel market on OD pair $(i, j)$ at the $k^{\text {th }}$ period can be expressed as

$$
Q_{i j}^{m}(k)=\min \left\{D_{i j}^{m}(k), S_{i j}^{m}(k)\right\} .
$$

Using formulas (4), (6), and (7), the multimodal travel market model on OD pair $(i, j)$ can be expressed as

$$
\left\{\begin{array}{l}
D_{i j}^{m}(k)=\alpha_{i j}^{m} X_{i j}^{m}(k)+\beta_{i j}^{m} P_{i j}^{m}(k)+\sum_{r \neq m} \varphi_{i j}^{r}\left[D_{i j}^{r}(k)-S_{i j}^{r}(k)\right], \\
S_{i j}^{m}(k)=\eta_{i j}^{m} Y_{i j}^{m}(k)+\sum_{i^{\prime} \neq i o r j^{\prime} \neq j} \vartheta_{i j-i^{\prime} j}^{m} S_{i^{\prime} j}^{m}(k), \\
Q_{i j}^{m}(k)=\min \left\{D_{i j}^{m}(k), S_{i j}^{m}(k)\right\} .
\end{array}\right.
$$

\section{Regulation Mechanism of Disequilibrium Multimodal Travel Market}

To regulate the supply-demand relationship of the disequilibrium multimodal travel market, this section attempts to seek the optimal price regulation law based on the multimodal travel market model by using the dynamic programming method.

3.1. Optimal Price Regulation Law. Let the total number of nodes in set $N$ be $\lambda$, the total number of travel modes in set $M_{i j}$ is $\ell_{i j}, D(k)=\left[D_{12}(k), \ldots, D_{1 \lambda}(k), D_{21}(k), \ldots, D_{\lambda(\lambda-1)}\right.$ $(k)]^{T}, X(k)=\left[X_{12}(k), \ldots, X_{1 \lambda}(k), X_{21}(k), \ldots, X_{\lambda(\lambda-1)}(k)\right]^{T}$, $P(k),=\left[P_{12}(k), \ldots, P_{1 \lambda}(k), P_{21}(k), \ldots, P_{\lambda(\lambda-1)}(k)\right]^{T}, \quad S(k)=$ $\left[S_{12}(k), \ldots, S_{1 \lambda}(k), S_{21}(k), \ldots, S_{\lambda(\lambda-1)}(k)\right]^{T}$, and $Y(k)=\left[Y_{12}\right.$ $\left.(k), \ldots, Y_{1 \lambda}(k), Y_{21}(k), \ldots, Y_{\lambda(\lambda-1)}(k)\right]^{T}$. Then, the effective demand and effective supply in the multimodal travel market model (8) can be rewritten as

$$
\begin{aligned}
& D(k)=\alpha X(k)+\beta P(k)+\varphi[D(k)-S(k)], \\
& S(k)=\eta Y(k)+\vartheta S(k),
\end{aligned}
$$

where 


$$
\begin{aligned}
& D_{i j}(k)=\left[D_{i j}^{1}(k), \ldots, D_{i j}^{\ell_{i j}}(k)\right] \text {, } \\
& S_{i j}(k)=\left[S_{i j}^{1}(k), \ldots, S_{i j}^{\ell_{i j}}(k)\right] \text {, } \\
& X_{i j}(k)=\left[X_{i j}^{1}(k), \ldots, X_{i j}^{\ell_{i j}}(k)\right] \text {, } \\
& Y_{i j}(k)=\left[Y_{i j}^{1}(k), \ldots, Y_{i j}^{\ell_{i j}}(k)\right] \text {, } \\
& P_{i j}(k)=\left[P_{i j}^{1}(k), \ldots, P_{i j}^{\ell_{i j}}(k)\right] \text {, } \\
& \alpha_{i j}=\operatorname{diag}\left(\alpha_{i j}^{1}, \ldots, \alpha_{i j}^{\ell_{i j}}\right), \quad \alpha=\operatorname{diag}\left(\alpha_{12}, \ldots, \alpha_{1 \lambda}, \alpha_{21}, \ldots, \alpha_{\lambda(\lambda-1)}\right) \text {, } \\
& \beta_{i j}=\operatorname{diag}\left(\beta_{i j}^{1}, \ldots, \beta_{i j}^{\ell_{i j}}\right), \quad \beta=\operatorname{diag}\left(\beta_{12}, \ldots, \beta_{1 \lambda}, \beta_{21}, \ldots, \beta_{\lambda(\lambda-1)}\right) \text {, } \\
& \eta_{i j}=\operatorname{diag}\left(\eta_{i j}^{1}, \ldots, \eta_{i j}^{\ell_{i j}}\right), \quad \eta=\operatorname{diag}\left(\eta_{12}, \ldots, \eta_{1 \lambda}, \eta_{21}, \ldots, \eta_{\lambda(\lambda-1)}\right), \\
& \varphi_{i j}=\left[\begin{array}{ccccc}
0 & \varphi_{i j}^{2} & \cdots & \varphi_{i j}^{\ell_{i j}-1} & \varphi_{i j}^{\ell_{i j}} \\
\varphi_{i j}^{1} & 0 & \cdots & \varphi_{i j}^{\ell_{i j}-1} & \varphi_{i j}^{\ell_{i j}} \\
\vdots & \vdots & \ddots & \vdots & \vdots \\
\varphi_{i j}^{1} & \varphi_{i j}^{2} & \cdots & 0 & \varphi_{i j}^{\ell_{i j}} \\
\varphi_{i j}^{1} & \varphi_{i j}^{2} & \cdots & \varphi_{i j}^{\ell_{i j}-1} & 0
\end{array}\right], \\
& \varphi=\operatorname{diag}\left(\varphi_{12}, \ldots, \varphi_{1 \lambda}, \varphi_{21}, \ldots, \varphi_{\lambda(\lambda-1)}\right) \text {, } \\
& \vartheta_{i j}^{i^{\prime \prime} j}=\left[\begin{array}{cccc}
\vartheta_{i j-i^{\prime} j}^{1} & 0 & \cdots & 0 \\
0 & \vartheta_{i j-i^{\prime \prime j}}^{2} & \cdots & 0 \\
\vdots & \vdots & \ddots & \vdots \\
0 & 0 & \cdots & \vartheta_{i j-i^{\prime \prime} j}^{\ell_{i^{\prime} j}}
\end{array}\right] \text {, } \\
& \vartheta=\left[\begin{array}{cccccc}
0 & \cdots & \vartheta_{12}^{1 \lambda} & \vartheta_{12}^{21} & \ldots & \vartheta_{12}^{\lambda(\lambda-1)} \\
\vdots & \ddots & \vdots & \vdots & \ddots & \vdots \\
\vartheta_{1 \lambda}^{12} & \cdots & 0 & \vartheta_{1 \lambda}^{21} & \ldots & \vartheta_{1 \lambda}^{\lambda(\lambda-1)} \\
\vartheta_{21}^{12} & \ldots & \vartheta_{21}^{1 \lambda} & 0 & \ldots & \vartheta_{21}^{\lambda(\lambda-1)} \\
\vdots & \ddots & \vdots & \vdots & \ddots & \vdots \\
\vartheta_{\lambda(\lambda-1)}^{12} & \cdots & \vartheta_{\lambda(\lambda-1)}^{1 \lambda} & \vartheta_{\lambda(\lambda-1)}^{21} & \cdots & 0
\end{array}\right] .
\end{aligned}
$$


Let $E=\operatorname{diag}(1,1, \ldots, 1), \quad|E-\varphi| \neq 0$ and $|E-\vartheta| \neq 0$. Formula (10) can be abbreviated as

$$
S(k)=(E-\vartheta)^{-1} \eta Y(k) .
$$

Applying formula (12) into (9), we have

$$
D(k)=(E-\varphi)^{-1}\left[\alpha X(k)+\beta P(k)-\varphi(E-\vartheta)^{-1} \eta Y(k)\right] .
$$

Let the supply-demand deviation $Z(k)=D(k)-S(k)$, we can obtain the following equation according to formulas (12) and (13).

$$
\begin{aligned}
Z(k)= & (E-\varphi)^{-1} \alpha X(k)-\left[(E-\varphi)^{-1} \varphi(E-\vartheta)^{-1} \eta\right. \\
& \left.+(E-\vartheta)^{-1} \eta\right] Y(k)+(E-\varphi)^{-1} \beta P(k) .
\end{aligned}
$$

For the convenience of analysis, we suppose that these exogenous variables remain unchanged; that is, $X(k+1)=$ $X(k)$ and $Y(k+1)=Y(k)$. Let the regulation amplitude of travel price $\Delta P(k)=P(k+1)-P(k), \quad U(k)=\Delta P(k)$, $A=E$, and $B=(E-\varphi)^{-1} \beta$; we thus have the following dynamic relational expression from formula (14).

$$
Z(k+1)=A Z(k)+B U(k) .
$$

To minimize the supply-demand deviation $Z(k)$ and avoid fierce market oscillation caused by excessive travel price regulation, we take the comprehensive minimization between the supply-demand deviation and the regulation amplitude of travel price as the objective function. Let $H_{0}$ be a symmetric positive definite matrix, $H=\operatorname{diag}\left(q_{12}, \ldots\right.$, $\left.q_{1 \lambda}, q_{21}, \ldots, q_{(\lambda \lambda-1)}\right), q_{i j}=\operatorname{diag}\left(q_{i j}^{1}, \ldots, q_{i j}^{\ell_{i j}}\right), q_{i j}^{1}, \ldots, q_{i j}^{\ell_{i j}}>0$, $R=\operatorname{diag}\left(\gamma_{12}, \ldots, \gamma_{1 \lambda}, \gamma_{21}, \ldots, \gamma_{\lambda(\lambda-1)}\right), \quad \gamma_{i j}=\operatorname{diag}\left(\gamma_{i j}^{1}, \ldots\right.$, $\left.\gamma_{i j}^{\ell_{i j}}\right)$, and $\gamma_{i j}^{1}, \ldots, \gamma_{i j}^{\ell_{i j}}>0$. Then, the objective function $J$ can be expressed as

$$
\begin{aligned}
\min J= & \frac{1}{2} Z^{T}(L) H_{0} Z(L) \\
& +\frac{1}{2} \sum_{k=0}^{L-1}\left[Z^{T}(k) H Z(k)+U^{T}(k) R U(k)\right] .
\end{aligned}
$$

By using the dynamic programming method (see Appendix) to solve formula (16), we obtain the optimal price regulation law of the disequilibrium multimodal travel market. It can be expressed as

$$
P(k+1)=P(k)-K Z(k),
$$

where $K=\left[B^{T} G B+R\right]^{-1} B^{T} G A, H_{0}=G$, and $G$ represents the positive definite solution of the following discrete Riccati equation.

$$
\begin{aligned}
G & =(A-B K)^{T} G(A-B K)+K^{T} R K+H \\
& =A^{T} G A-A^{T} G B\left(B^{T} G B+R\right)^{-1} B^{T} G A+H .
\end{aligned}
$$

\subsection{Stability Analysis}

Theorem 1. For the optimal regulation problem (16), if $k$ is finite, then there exists a unique optimal price regulation law of discrete system (15).

Proof. We first prove the existence of the optimal price regulation law $U^{*}(k)$. Because $A, B, R$, and $H$ are constant matrices, then $G$ is unique according to formula (18); thus, $K$ is also unique. Evidently, there exists at least one optimal price regulation law $U^{*}(k)$ in discrete system (15).

Second, we prove the uniqueness of the optimal price regulation law $U^{*}(k)$. Suppose that there exist two different optimal price regulation laws, $U_{1}^{*}(k)$ and $U_{2}^{*}(k)$, in discrete system (15). From the uniqueness of $K$, we know the following:

$$
\begin{aligned}
& U_{1}^{*}(k)=-K Z_{1}(k), \\
& U_{2}^{*}(k)=-K Z_{2}(k) .
\end{aligned}
$$

Applying formulas (19) and (20) into (15), respectively, we have

$$
\begin{aligned}
& Z_{1}^{*}(k+1)=(A-B K) Z_{1}(k), \\
& Z_{2}^{*}(k+1)=(A-B K) Z_{2}(k) .
\end{aligned}
$$

Because $Z_{1}^{*}$ and $Z_{2}^{*}$ are solutions of the same discrete system with the same initial condition, we know that $Z_{1}^{*}(k+$ $1)=Z_{2}^{*}(k+1)$ from formula (21) and (22); that is, $U_{1}^{*}(k)=U_{2}^{*}(k)$. Hence, the optimal regulation problem (16) has a unique optimal price regulation law $U^{*}(k)$.

Theorem 2. If the optimal regulation problem (16) of discrete system (15) has a unique optimal price regulation law $U^{*}(k)$, then the optimal price regulation law $U^{*}(k)$ is asymptotically stable.

Proof. We first introduce the stability criterion of discrete system (Tian [33]).

3.2.1. Stability Criterion of Discrete System. If there exists a discrete function $V(Z(k))$ for discrete system $(15), V(Z(k))$ satisfies the conditions,

(1) $\left.V(Z(k))\right|_{Z(k)=0}=0$ and $\left.V(Z(k))\right|_{Z(k) \neq 0}>0$,

(2) $\Delta V(Z(k))=V(Z(k+1))-V(Z(k))<0$,

then the optimal price regulation law $U^{*}(k)$ is asymptotically stable.

Constructing a discrete function $V(Z(k))$, it can be written as

$$
V(Z(k))=Z^{T}(k) G Z(k)
$$

According to the positive definite solution $G$ and formula (23), we know that $V(Z(k))$ satisfies condition (1) in the stability criterion of discrete system, and we only need to prove that $\Delta V(Z(k))<0$. We know 


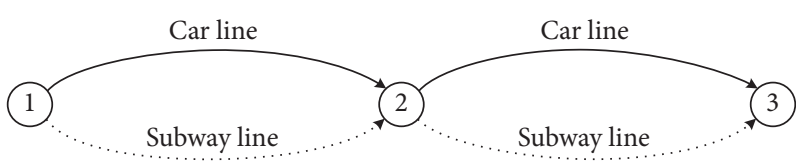

FIgURE 1: Test road network.

$$
\Delta V(Z(k))=Z^{T}(k)\left[(A-B K)^{T} G(A-B K)-G\right] Z(k) .
$$

According to formula (18), formula (24) can be rewritten as

$$
\Delta V(Z(k))=-Z^{T}(k)\left[H+K^{T} R K\right] Z(k) .
$$

Because $H, K$, and $R$ are positive definite matrices, we know that $H+K^{T} R K>0$; thus, $\Delta V(Z(k))<0$. Therefore, the optimal price regulation law $U^{*}(k)$ is asymptotically stable.

$$
\begin{aligned}
& \left\{\begin{array}{l}
D_{12}^{c_{12}}(k)=300-2 P_{12}^{c_{12}}(k)+0.2 \times\left[D_{12}^{s_{12}}(k)-S_{12}^{s_{12}}(k)\right] \\
S_{12}^{c_{12}}(k)=0.4 \times 500+0.2 \times S_{13}^{c_{13}}(k) \\
Q_{12}^{c_{12}}(k)=\min \left\{D_{12}^{c_{12}}(k), S_{12}^{c_{12}}(k)\right\}
\end{array}\right. \\
& \left\{\begin{array}{l}
D_{13}^{c_{13}}(k)=350-2 P_{13}^{c_{13}}(k)+0.2 \times\left[D_{13}^{s_{13}}(k)-S_{13}^{s_{13}}(k)\right] \\
S_{13}^{c_{13}}(k)=0.4 \times 500+0.2 \times S_{12}^{c_{12}}(k) \\
Q_{13}^{c_{13}}(k)=\min \left\{D_{13}^{c_{13}}(k), S_{13}^{c_{13}}(k)\right\}
\end{array}\right.
\end{aligned}
$$

In the effective demand $D_{12}^{c_{12}}$ of driverless car travel market on OD pair $(1,2), X_{12}^{c_{12}}=300$ represents the total travel demand of driverless car on OD pair $(1,2)$ at the $k^{\text {th }}$ hour, $\alpha_{12}^{c_{12}}=1$ represents the influence of $X_{12}^{c_{12}}$ on $D_{12}^{c_{12}}, P_{12}^{c_{12}}$ represents the driverless car travel price on OD pair $(1,2)$ at the $k^{\text {th }}$ hour, $\beta_{12}^{c_{12}}=-2$ represents the sensitivity of $P_{12}^{c_{12}}$ on $D_{12}^{c_{12}}, \quad D_{12}^{s_{12}}-S_{12}^{s_{12}}$ represents the effective supply-demand difference of driverless subway on OD pair $(1,2)$ at the $k^{\text {th }}$ hour, and $\varphi_{12}^{c_{12}}=0.2$ represents the sensitivity of $D_{12}^{s_{12}}-S_{12}^{s_{12}}$ on $D_{12}^{c_{12}}$.

In the effective supply $S_{12}^{c_{12}}$ of driverless car travel market on OD pair $(1,2), Y_{12}^{c_{12}}=500$ represents the total supply on OD pair $(1,2)$ at the $k^{\text {th }}$ hour, $\eta_{12}^{c_{12}}=0.4$ represents the ratio of the supply meet driverless car travel demand to the total supply on OD pair $(1,2), S_{13}^{c_{13}}$ represents the effective supply of driverless car travel market on OD pair $(1,3)$, and $\vartheta_{12-13}^{c_{12}}$ represents the influence of $S_{13}^{c_{13}}$ on $S_{12}^{c_{12}}$.

$D_{12}^{s_{12}}, S_{12}^{s_{12}}, D_{13}^{c_{13}}, S_{13}^{c_{13}}, D_{13}^{s_{13}}$, and $S_{13}^{s_{13}}$ are similar to the definitions of the above variables in the models (26) and (27).

4.2. Model Calculation and Result Analysis. Suppose that $H=\operatorname{diag}(1,1,1,1), R=\operatorname{diag}(8,800,8,600)$, the regulation period $k \in[0,24]$, the regulation step $\Delta k=1$, the initial

\section{Numerical Examples}

4.1. Test Road Network and Market Model. To validate the proposed optimal price regulation law, we need to establish the corresponding relationship between travel price in the theoretical model and specific traffic management measures. Hence, we suppose that the test road network includes OD pairs $(1,2)$ and $(1,3)$, driverless car, and driverless subway in Figure 1, in which the travel price of driverless car and driverless subway are regulated by road congestion fee CF and subway fare SF, respectively.

Suppose that the driverless car travel price $P_{i j}^{c_{i j}}(k) \underset{=}{=} P_{i j}^{c_{i j}}(0)+\mathrm{CF}_{i j}^{c_{i j}}(k)$ and the driverless subway travel price $P_{i j}^{s_{i j}}(k)=\mathrm{SF}_{i j}^{s_{i j}}(k)$; we construct the following multimodal travel market model on OD pairs $(1,2)$ and $(1,3)$.

$$
\begin{aligned}
& D_{12}^{s_{12}}(k)=300-25 P_{12}^{s_{12}}(k)+0.2 \times\left[D_{12}^{c_{12}}(k)-S_{12}^{c_{12}}(k)\right] \\
& S_{12}^{s_{12}}(k)=0.8 \times 300+0.1 \times S_{13}^{s_{13}}(k), \\
& Q_{12}^{s_{12}}(k)=\min \left\{D_{12}^{s_{12}}(k), S_{12}^{s_{12}}(k)\right\} \\
& D_{13}^{s_{13}}(k)=360-30 P_{13}^{s_{13}}(k)+0.2 \times\left[D_{13}^{c_{13}}(k)-S_{13}^{c_{13}}(k)\right] \\
& S_{13}^{s_{13}}(k)=0.8 \times 300+0.1 \times S_{12}^{s_{12}}(k), \\
& Q_{13}^{s_{13}}(k)=\min \left\{D_{13}^{s_{13}}(k), S_{13}^{s_{13}}(k)\right\}
\end{aligned}
$$

travel price $P_{12}^{c_{12}}(0)=20, \quad P_{12}^{s_{12}}(0)=2, \quad P_{13}^{c_{13}}(0)=30$, and $P_{13}^{s_{13}}(0)=4$. The results of calculation are presented in Figure 2 and Table 1.

As shown in Figure 2, the driverless car travel markets on OD pairs $(1,2)$ and $(1,3)$ at the beginning are in the state of overdemand, and the driverless subway travel markets are in the state of oversupply. After a certain number of regulation periods, the supply-demand relationship of the driverless car and driverless subway travel markets on OD pairs $(1,2)$ and $(1,3)$ reaches the equilibrium state. The results indicate that the proposed optimal price regulation law can significantly reduce the supply-demand deviation of the multimodal travel market and avoid the agitation of travel market caused by excessive price regulation.

The optimal price regulation results of driverless car and driverless subway travel markets on OD pairs $(1,2)$ and $(1,3)$ within $0-9$ periods are listed in Table 1 . Combining the results of Figure 2 and Table 1 reveals that increasing the road congestion fee and reducing the subway fare will motivate some travelers who plan to travel by driverless car to take the driverless subway or give up their travel plans. Ultimately, it makes the demand of driverless subway travel market increase, makes the demand of driverless car travel market decrease, and 


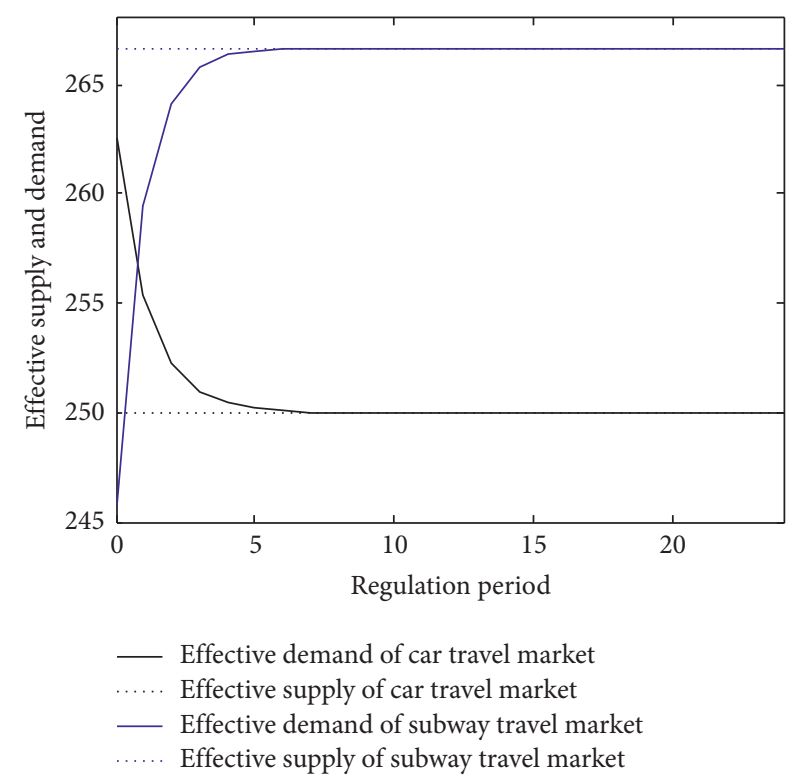

(a)

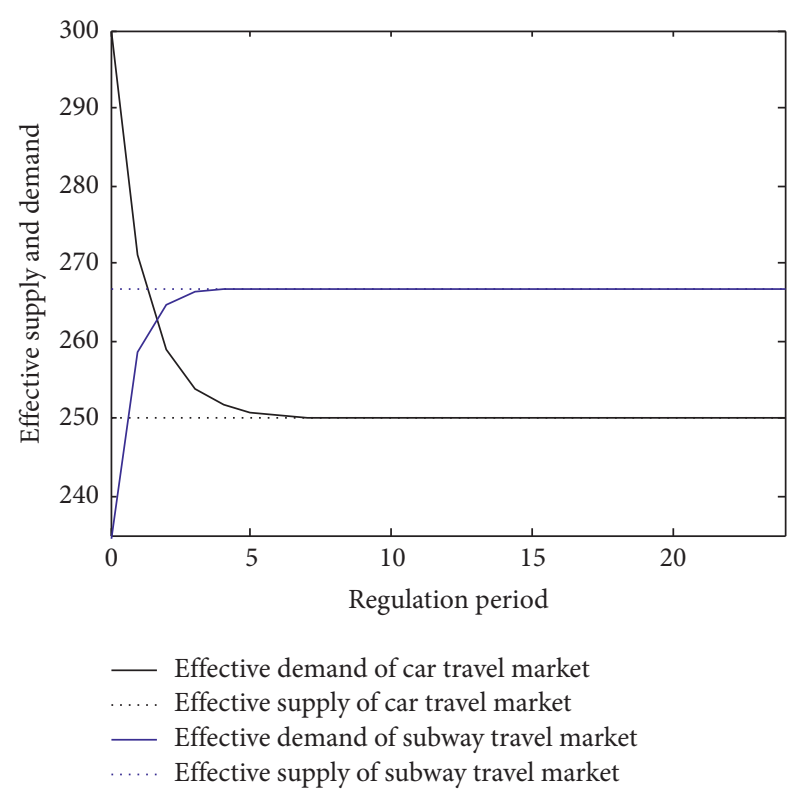

(b)

FIGURE 2: Regulation process of effective supply and demand in the multimodal travel market. (a) OD pair $(1,2)$. (b) OD pair $(1,3)$.

TABLE 1: Optimal price regulation results.

\begin{tabular}{lcccc}
\hline \multirow{2}{*}{ Regulation period $(\mathrm{h})$} & \multicolumn{2}{c}{ OD pair $(1,2)$} & \multicolumn{2}{c}{ OD pair $(1,3)$} \\
\hline 0 & Congestion fee & Subway fare & Congestion fee & Subway fare \\
1 & 0 & 2 & 11.5146 & 4 \\
2 & 2.8786 & 1.5654 & 16.3999 & 3.3279 \\
3 & 4.1000 & 1.4141 & 18.4726 & 3.1640 \\
4 & 4.6181 & 1.3615 & 19.3520 & 3.1240 \\
5 & 4.8380 & 1.3431 & 19.7251 & 3.1143 \\
6 & 4.9313 & 1.3367 & 19.8833 & 3.1119 \\
7 & 4.9708 & 1.3345 & 19.9505 & 3.1113 \\
8 & 4.9876 & 1.3337 & 19.9790 & 3.1111 \\
9 & 4.9948 & 1.3335 & 19.9911 & 3.1111 \\
\hline
\end{tabular}

finally achieves market equilibrium. The results show that the optimal price regulation law can help travelers to choose a reasonable travel mode to travel.

\section{Conclusions}

In this study, by taking each type of driverless travel modes on each OD pair as a travel market and taking the driving time, driving cost, and perceived traffic service quality as the travel price, we established a multimodal travel market model. Aiming at achieving the minimum supply-demand deviation as well as the minimum amplitude of price regulation, we proposed an optimal pricequantity law of the disequilibrium multimodal travel market and analyzed the stability of the optimal price regulation law. Through a numerical example, the application of the optimal price regulation law in urban multimodal traffic management was studied. The results indicated that the optimal price regulation law can effectively reduce the supply-demand deviation of the multimodal travel market, avoid the agitation of the travel market caused by excessive price regulation, and achieve market equilibrium.

This study can help travelers choose a reasonable travel mode to travel and promote the development of urban traffic demand management methods. In a future study, we plan to consider the mixed traffic scenario of manual and driverless vehicle, the effects of the travel price on the notional supply in the long period, and the influence of the expected trading volume on traveler' travel mode choice.

\section{Appendix}

The processes of solving formula (16) by the dynamic programming method are as follows. Let the $k+1$ level decision process be a process from $k+1$ level to the terminal state through $L-k-1$ level decision-making. Then, the performance index $J_{k+1}$ of the $k+1$ level decision process can be expressed as 


$$
\begin{aligned}
J_{k+1}= & \frac{1}{2} Z^{T}(L) H_{0} Z(L) \\
& +\frac{1}{2} \sum_{\tau=k+1}^{L-1}\left[Z^{T}(\tau) H Z(\tau)+U^{T}(\tau) R U(\tau)\right] .
\end{aligned}
$$

Hence, the performance index $J_{k}$ of the $k$ level decision process can be expressed as

$$
J_{k}=J_{k+1}+\frac{1}{2}\left[Z^{T}(k) H Z(k)+U^{T}(k) R U(k)\right] .
$$

According to Bellman's principle of optimality (Gross [34]), the optimal performance index of formula (29) can be expressed as

$$
J_{k}^{*}=\min _{U(k)}\left\{J_{k+1}^{*}+\frac{1}{2}\left[Z^{T}(k) H Z(k)+U^{T}(k) R U(k)\right]\right\} .
$$

Let $G$ be the symmetric matrix and $J_{k}^{*}=1 / 2 Z^{T}(k) G Z(k)$. Then, we have

$$
J_{k+1}^{*}=\frac{1}{2} Z^{T}(k+1) G Z(k+1) .
$$

By applying formula (15) into (31), we obtain

$$
J_{k+1}^{*}=\frac{1}{2}[A Z(k)+B U(k)]^{T} G[A Z(k)+B U(k)] .
$$

By applying formula (32) into (30), we obtain

$$
\begin{aligned}
J_{k}^{*}= & \min _{U(k)}\left\{Z^{T}(k)\left[H+A^{T} G A\right] Z(k)+2 U^{T}(k) B^{T} G A Z(k)\right. \\
& \left.+U^{T}(k)\left[B^{T} G B+R\right] U(k)\right\} .
\end{aligned}
$$

We also know that the regulation variable $U(k)$ has no effect on the current state $Z(k)$ from formula (15). Therefore, we have

$$
\frac{\partial J_{k}^{*}}{\partial U(k)}=2 B^{T} G A Z(k)+2\left[B^{T} G B+R\right] U(k)=0 .
$$

According to formula (34), we obtain the optimal price regulation law of the disequilibrium multimodal travel market, which can be expressed as

$$
U^{*}(k)=-K Z(k) .
$$

Equivalently,

$$
P(k+1)=P(k)-K Z(k) .
$$

\section{Data Availability}

The data used to support the findings of this study are available from the corresponding author upon request.

\section{Conflicts of Interest}

The authors declare that there are no conflicts of interest regarding the publication of this paper.

\section{Acknowledgments}

This research work was supported by the National Natural Science Foundation of China (Grant nos. 61561016 and 61861008), National Key R\&D Program of China (Grant no. 2018YFB0505103), Hunan Provincial Natural Science Foundation of China (Grant no. 2019JJ50210), Scientific Research Foundation of Hunan Provincial Education Department (Grant no. 19B234), and Foundation from the Guangxi Zhuang Autonomous Region (Grant nos. AC16380014, AA17202048, and AA17202033).

\section{References}

[1] M. M. Morando, Q. Y. Tian, L. T. Truong, and H. L. Vu, "Studying the safety impact of autonomous vehicles using simulation-based surrogate safety measures," Journal of Advanced Transportation, vol. 2018, p. 11, 2018.

[2] L. Ye and T. Yamamoto, "Impact of dedicated lanes for connected and autonomous vehicle on traffic flow throughput," Physica A: Statistical Mechanics and Its Applications, vol. 512, pp. 588-597, 2018.

[3] P. M. Smolnicki and J. Sołtys, "Driverless mobility: the impact on metropolitan spatial structures," Procedia Engineering, vol. 161, pp. 2184-2190, 2018.

[4] W. Zhang, S. Guhathakurta, J. Fang, and G. Zhang, "Exploring the impact of shared autonomous vehicles on urban parking demand: an agent-based simulation approach," Sustainable Cities and Society, vol. 19, pp. 34-45, 2015.

[5] J. Kamel, R. Vosooghi, J. Puchinger, F. Ksontini, and G. Sirin, "Exploring the impact of user preferences on shared autonomous vehicle modal split: a multi-agent simulation approach," Transportation Research Procedia, vol. 37, pp. 115-122, 2019.

[6] Z. Yi, J. Smart, and M. Shirk, "Energy impact evaluation for eco-routing and charging of autonomous electric vehicle fleet: ambient temperature consideration," Transportation Research Part C: Emerging Technologies, vol. 89, pp. 344-363, 2018.

[7] A. T. Moreno, A. Michalski, C. Llorca, and R. Moeckel, "Shared autonomous vehicles effect on vehicle-km traveled and average trip duration," Journal of Advanced Transportation, vol. 2018, Article ID 8969353, 10 pages, 2018.

[8] Z. Liu and Z. Song, "Strategic planning of dedicated autonomous vehicle lanes and autonomous vehicle/toll lanes in transportation networks," Transportation Research Part C: Emerging Technologies, vol. 106, pp. 381-403, 2019.

[9] C. Xia, X. Jin, L. Kong, C. Xu, and P. Zeng, "Lane scheduling around crossroads for edge computing based autonomous driving," Journal of Systems Architecture, vol. 95, pp. 1-8, 2019.

[10] J. M. L. Domínguez and T. J. M. Sanguino, "Design, modelling, and implementation of a fuzzy controller for an intelligent road signaling system," Complexity, vol. 2018, Article ID 1849527, 14 pages, 2018.

[11] N. Jiang, "Optimal signal design for mixed equilibrium networks with autonomous and regular vehicles," Journal of Advanced Transportation, vol. 2017, Article ID 5649823, 13 pages, 2017.

[12] M. Tajalli and A. Hajbabaie, "Distributed optimization and coordination algorithms for dynamic speed optimization of connected and autonomous vehicles in urban street networks," Transportation Research Part C: Emerging Technologies, vol. 95, pp. 497-515, 2018. 
[13] D. Li, Y. Zhao, P. Ranjitkar, H. Zhao, and Q. Bai, "Hybrid approach for variable speed limit implementation and application to mixed traffic conditions with connected autonomous vehicles," IET Intelligent Transport Systems, vol. 12, no. 5, pp. 327-334, 2018.

[14] M. Nourinejad, S. Bahrami, and M. J. Roorda, "Designing parking facilities for autonomous vehicles," Transportation Research Part B: Methodological, vol. 109, pp. 110-127, 2018.

[15] R. Estepa, A. Estepa, J. Wideberg, M. Jonasson, and A. Stensson-Trigell, "More effective use of urban space by autonomous double parking," Journal of Advanced Transportation, vol. 2017, Article ID 8426946, 10 pages, 2017.

[16] S. Yamashita and K. Takami, "Autonomous, distributed parking lot vacancy management using intervehicle communication," International Journal of Vehicular Technology, vol. 2014, Article ID 647487, 9 pages, 2014.

[17] B. Wang, S. A. Ordonez Medina, and P. J. Fourie, "Comparing parking strategies of autonomous transit on demand with varying transport demand," Procedia Computer Science, vol. 151, pp. 814-819, 2019.

[18] G. Leich and J. Bischoff, "Should autonomous shared taxis replace buses? a simulation study," Transportation Research Procedia, vol. 41, pp. 450-460, 2019.

[19] R. Abe, "Introducing autonomous buses and taxis: quantifying the potential benefits in Japanese transportation systems," Transportation Research Part A: Policy and Practice, vol. 126, pp. 94-113, 2019.

[20] Z. Cao and A. Ceder, "Autonomous shuttle bus service timetabling and vehicle scheduling using skip-stop tactic," Transportation Research Part C: Emerging Technologies, vol. 102, pp. 370-395, 2019.

[21] L. Tong, L. Zhou, J. Liu, and X. Zhou, "Customized bus service design for jointly optimizing passenger-to-vehicle assignment and vehicle routing," Transportation Research Part C: Emerging Technologies, vol. 85, pp. 451-475, 2017.

[22] L. Ye and T. Yamamoto, "Evaluating the impact of connected and autonomous vehicles on traffic safety," Physica A: Statistical Mechanics and Its Applications, vol. 526, Article ID 121009, 12 pages, 2019.

[23] N. Virdi, H. Grzybowska, S. T. Waller, and V. Dixit, “A safety assessment of mixed fleets with connected and autonomous vehicles using the surrogate safety assessment module," Accident Analysis \& Prevention, vol. 131, pp. 95-111, 2019.

[24] H. Prakken, "On the problem of making autonomous vehicles conform to traffic law," Artificial Intelligence and Law, vol. 25, no. 3, pp. 341-363, 2017.

[25] C. Bartolini, T. Tettamanti, and I. Varga, "Critical features of autonomous road transport from the perspective of technological regulation and law," Transportation Research Procedia, vol. 27, pp. pp791-798, 2017.

[26] P. Wagner, Traffic Control and Traffic Management in a Transportation System with Autonomous Vehicles, Springer, Berlin, Germany, 2016.

[27] T. Tettamanti, A. Mohammadi, H. Asadi, and I. Varga, "A two-level urban traffic control for autonomous vehicles to improve network-wide performance," Transportation Research Procedia, vol. 27, pp. 913-920, 2017.

[28] R. Kitamura, S. Fujii, and E. I. Pas, "Time-use data, analysis and modeling: toward the next generation of transportation planning methodologies," Transport Policy, vol. 4, no. 4, pp. 225-235, 1997.

[29] R. Brideges, Our Driverless Future: Heaven or Hell?, Understanding Disruption, Inc., Great Britain, 2018.
[30] Y. Ye and H. Wang, "Optimal design of transportation networks with automated vehicle links and congestion pricing," Journal of Advanced Transportation, vol. 2018, Article ID 3435720, 12 pages, 2018.

[31] X. Zhang, W. Liu, S. T. Waller, and Y. Yin, "Modelling and managing the integrated morning-evening commuting and parking patterns under the fully autonomous vehicle environment," Transportation Research Part B: Methodological, vol. 128, pp. 380-407, 2019.

[32] F. X. Browne, "Sluggish quantity adjustment in a non-clearing market-a disequilibrium econometric application to the loan market," Journal of Applied Econometrics, vol. 2, no. 4, pp. 335-349, 1987.

[33] X. G. Tian, "On going further into the extended stability theorem for discrete system," Journal of Tianjin University, vol. 28, no. 3, pp. 348-353, 1995.

[34] E. Gross, "On the Bellman's principle of optimality," Physica A: Statistical Mechanics and Its Applications, vol. 462, pp. 217-221, 2016. 